\title{
The short-term outcomes of minimally invasive decompression surgery in patients with lumbar ossification or calcification of the ligamentum flavum
}

\author{
Koji Tamai, MD, ${ }^{1}$ Kunikazu Kaneda, MD, ${ }^{2}$ Masayoshi Iwamae, MD, ${ }^{1}$ Hidetomi Terai, MD, \\ Hiroshi Katsuda, MD, ${ }^{2}$ Nagakazu Shimada, MD, ${ }^{2}$ and Hiroaki Nakamura, MD1 \\ 1Department of Orthopaedic Surgery, Osaka City University Graduate School of Medicine; and 2Department of Orthopaedic \\ Surgery, Shimada Hospital, Osaka, Japan
}

\begin{abstract}
OBJECTIVE Although minimally invasive endoscopic surgery techniques are established standard treatment choices for various degenerative conditions of the lumbar spine, the surgical indications of such techniques for specific cases, such as segments with ossification of the ligamentum flavum (OLF) or calcification of the ligamentum flavum (CLF), remain under investigation. Therefore, the authors aimed to demonstrate the short-term outcomes of minimally invasive endoscopic surgery in patients with degenerative lumbar disease with CLF or OLF.

METHODS This is a retrospective cohort study including consecutive patients who underwent microendoscopic posterior decompression at the authors' institution, where the presence of OLF and CLF did not influence the surgical indication. Fifty-nine patients with OLF and 39 patients with CLF on preoperative CT were identified from the database. Subsequently, two matched control groups (one each matched to the OLF and CLF groups) were created using propensity scores to adjust for age, sex, preoperative Japanese Orthopaedic Association (JOA) score and Oswestry Disability Index, and diagnosis. The background, surgical outcomes, and changes in clinical scores were compared between the matched groups. If there was a significant difference in the improvement of clinical scores, a multivariate linear regression model was applied.
\end{abstract}

RESULTS On performing univariate analysis, patients with OLF were found to have a higher body mass index (MannWhitney U-test, $p=0.001$ ), higher incidence of preoperative motor weakness (chi-square test, $p=0.019$ ), longer operative time (Mann-Whitney U-test, $p<0.001$ ), and lower improvement in the JOA score (mixed-effects model, $p=0.023$ ) than the matched controls. On performing multivariate analysis, the presence of OLF was identified as an independent variable associated with a poor recovery rate based on the JOA score (multivariate linear regression, $p<0.001$ ). In contrast, there were no significant differences between patients with CLF and their matched controls in terms of preoperative and surgical data and postoperative improvements in clinical scores.

CONCLUSIONS Although the perioperative surgical outcomes, including the surgical complications, and the in-hospital period did not significantly differ, the short-term improvement in the JOA score was significantly lower in patients with degenerative lumbar disease accompanied by OLF than in the patients from the matched control group. In contrast, there were no significant differences in the short-term improvement in clinical scores and perioperative outcomes between patients with CLF and their matched control group. Thus, the surgical indications of minimally invasive posterior decompression for patients with CLF can be the same as those for patients without CLF; however, the indications for patients with OLF should be further investigated in future studies, including the other surgical methods.

https://thejns.org/doi/abs/10.3171/2020.6.SPINE20946

KEYWORDS ossification of ligamentum flavum; calcification of ligamentum flavum; minimally invasive surgery; microendscopic surgery; lumbar degenerative disease; surgical outcome; Oswestry Disability Index; Japanese Orthopaedic Association score

ABBREVIATIONS BMI = body mass index; CLF = calcification of the ligamentum flavum; JOA = Japanese Orthopaedic Association; ODI = Oswestry Disability Index; OLF $=$ ossification of the ligamentum flavum. SUBMITTED May 25, 2020. ACCEPTED June 29, 2020.

INCLUDE WHEN CITING Published online November 6, 2020; DOI: 10.3171/2020.6.SPINE20946. 
$\mathrm{O}$ SSIFICATION of the ligamentum flavum (OLF) and calcification of the ligamentum flavum (CLF) of the lumbar spine are relatively rare conditions. The incidence of OLF, which involves replacement of the ligamentum flavum by mature lamellar bone, ${ }^{1,2}$ has been reported to be up to $4 \%$ among the general population. ${ }^{3}$ Although the most common OLF lesion is at the thoracic level, it has been reported that $10 \%$ of all OLF cases involve the lumbar spine. ${ }^{4,5}$ Meanwhile, CLF, which is considered to be one of the degenerative changes of the ligament, occurs mainly in the ligamentum flavum of the cervical spine rather than in the lumbar spine. ${ }^{6}$ However, previous reports have demonstrated that CLF correlates with lumbar spinal canal stenosis. ${ }^{7,8}$

Minimally invasive endoscopic surgery, especially endoscope-assisted posterior decompression surgery, lessens tissue damage, reduces postoperative pain, shortens the hospital stay, and allows quick rehabilitation. ${ }^{9}$ Hence, such techniques are established standard treatment choices for various degenerative conditions of the lumbar spine, including lumbar disc herniation and lumbar spinal stenosis, with good outcomes. ${ }^{10,11}$ However, the surgical indications of these minimally invasive techniques for specific cases, such as segments with CLF or OLF, remain under investigation. ${ }^{12}$

Many factors impact surgical outcomes, including patient age, the severity of preoperative neurological symptoms, and diagnosis. ${ }^{13,14}$ Because of inherent limitations in observational data sets, propensity score matching, which is a statistical procedure enabling the selection of matched control groups using logistic regression analysis, was established to reduce the effects of potential confounding factors. ${ }^{15,16}$ Therefore, by using control groups with adjustment for basic demographics via propensity score matching, the present study aimed to demonstrate the surgical outcomes of minimally invasive endoscopic surgery for patients with degenerative lumbar disease accompanied by OLF or CLF.

\section{Methods}

\section{Study Design and Ethics}

We conducted a retrospective cohort study. All study participants provided informed consent, and the study protocol was approved by the institutional review board of Shimada Hospital. No funds were received in support of this work. No benefits in any form have been or will be received from a commercial party related directly or indirectly to the subject of this paper. All methods were performed in accordance with the Declaration of Helsinki and the Ethical Guidelines for Medical and Health Research Involving Human Subjects in Japan.

\section{Patient Population}

We reviewed the medical records of 4829 consecutive patients who underwent microendoscopic posterior decompression at Shimada Hospital between 2005 and 2019 and were followed up for at least 3 months postoperatively (1692 women, 3137 men; mean age [ $\pm \mathrm{SD}]$ at surgery 56.3 \pm 17.7 years). The diagnoses included lumbar disc herniation ( $\mathrm{n}=2243)$, lumbar spinal stenosis $(\mathrm{n}=1983)$, foraminal stenosis $(\mathrm{n}=131)$, and others such as far-out syndrome or facet cyst $(n=513)$.

\section{Surgical Criteria}

Posterior endoscopic decompression was indicated in patients who had neurogenic claudication or radicular pain with associated neurological signs, who also had stenosis or herniation on MRI at a level that explained their symptoms, and who did not improve in spite of adequate conservative treatment for at least 3 months. Exclusion criteria were as follows: spondylolisthesis of more than grade 2, spondylolytic spondylolisthesis, and degenerative lumbar scoliosis with a Cobb angle $>20^{\circ}$. The presence of OLF and CLF did not influence the surgical indication and surgical level in the current case series.

\section{Perioperative Clinical Care}

All patients were treated according to the standardized care pathways of our institution. Decompression surgery was performed under general anesthesia as previously reported. ${ }^{17}$ All patients were allowed to sit and walk with a soft brace on the day after surgery. The standard care protocol includes the routine use of celecoxib $200 \mathrm{mg}$ per day as a painkiller for 7 days after surgery, and allowed the use of additional painkillers such as acetaminophen, opioids, or nonsteroidal antiinflammatory drugs (oral or intravenous administration) as needed. All patients underwent lumbar MRI at 3 days postoperatively and were recommended for hospital discharge at 6 or 7 days postoperatively.

\section{Definition of OLF and CLF}

The presence of OLF and CLF was evaluated using axial images from the preoperative CT scan. All lumbar CT scans were obtained sequentially at a $0.5-\mathrm{mm}$ thickness, and the images were obtained with the patient supine, using a 64-channel CT scanner (OptimaCT660 pro advance; General Electric Co.). Based on previous reports, the appearance of OLF was defined as ossified masses arising from the lamina and/or distinctive ossified plaques within the ligamentum flavum (Fig. 1A and B). ${ }^{1,18}$ Meanwhile, the appearance of CLF was defined as oval calcified nodules seen within the ligamentum flavum and not continuous with the lamina (Fig. $1 \mathrm{C}$ and D). ${ }^{19}$ Patients with apparent OLF or CLF at the surgery levels were defined as having OLF or CLF. Two observers (K.T. and M.I.) independently evaluated all CT images from L1-2 to L5-S1; any differences in the evaluation were settled by consensus of the two observers. The kappa coefficient within 1 observer after a 1-month interval was $0.810(\mathrm{p}<$ 0.001 ), and that between the two observers was 0.711 ( $\mathrm{p}$ $<0.001)$.

\section{Preoperative Data}

Data regarding the patients' age at surgery, sex, height, weight, body mass index (BMI), and comorbidities (diabetes mellitus, hypertension, cardiac disease, respiratory disease, and cerebrovascular disease) were collected from the medical records. In terms of preoperative symptoms, data regarding motor weakness and bowel and bladder syndrome were collected.

\section{Surgical Data}

Surgical data including the total operative time and the 

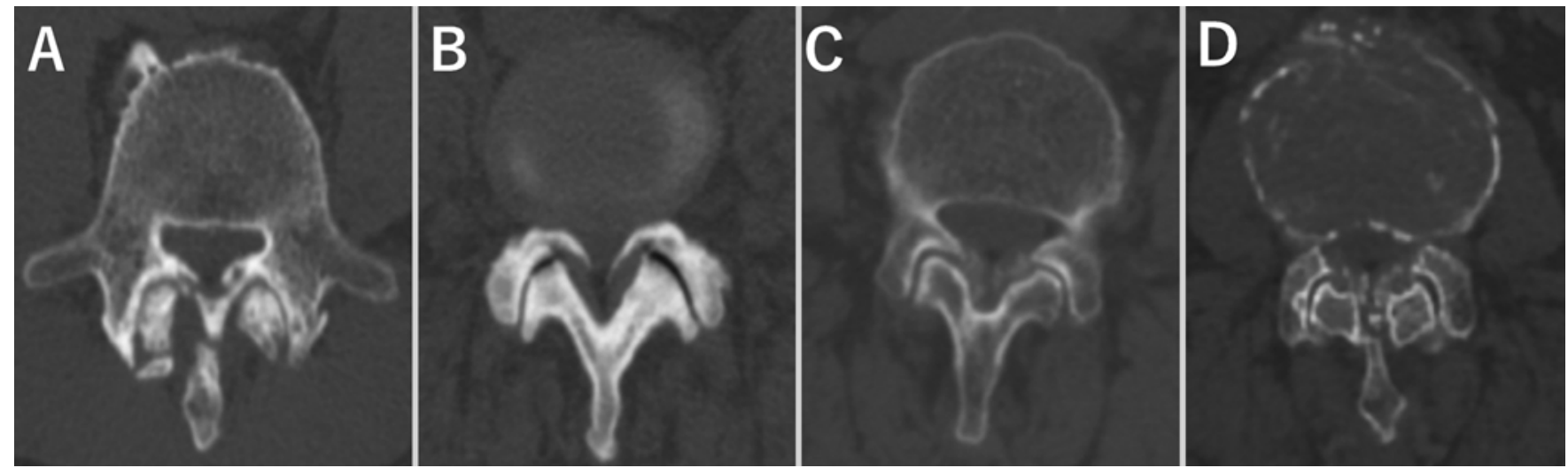

FIG. 1. Illustrative axial images of OLF and CLF. A: A 65-year-old man with OLF at the L5-S1 level. B: A 46-year-old woman with OLF at the L3-4 level. C: A 75-year-old woman with CLF at the L4-5 level. D: An 83-year-old woman with CLF at the L3-4 level.

number of surgical segments and dural punctures were collected. The operative time per segment was calculated by dividing the total operative time by the number of surgical segments. Postoperative surgical data, including neurological deterioration, in-hospital period, the use of additional painkillers, and the presence of hematoma on MRI, were collected. When a patient used a painkiller in addition to the routinely prescribed celecoxib, the number of times of use was recorded. Postoperative hematoma was assessed using the MRI sequence obtained at 3 days postoperatively. When the dura mater at the level of decompression surgery was compressed by a hematoma, the case was recorded as hematoma positive.

\section{Clinical Scores}

The Japanese Orthopaedic Association (JOA) score for degenerative lumbar disease and the Oswestry Disability Index $(\mathrm{ODI})^{20,21}$ were collected preoperatively and at 3 months postoperatively. The recovery rate was calculated as follows: recovery rate $=100 \times$ (postoperative score preoperative score)/(full score - preoperative score). ${ }^{22}$

\section{Study Design and Statistical Analysis}

All patients were first classified into three groups: the OLF group, the CLF group, and the control group (patients without OLF or CLF). In overall comparisons, the average age, average preoperative JOA score, ratio of males to females, and diagnosis were compared between these three groups by using the 1-way ANOVA or chisquare test, with the Tukey test or residual analysis as the post hoc analysis. The result of the residual analysis was considered to indicate $\mathrm{p}<0.05$ when all variables had $|r|>1.96$, in accordance with the Haberman method. ${ }^{23}$ Subsequently, two matched control groups, one matched to the patients in the OLF group and the other matched to the patients in the CLF group, were created using propensity score matching. To estimate the propensity score, we fitted a logistic regression model using the patient's age, sex, preoperative JOA score, and diagnosis. The nearest-neighbor matching procedure was used, with the restriction that the matched propensities had to be within
0.0001 units of each other. To identify the characteristics associated with OLF or CLF, preoperative and surgical data were compared between the OLF or CLF group and their matched control group, using the chi-square test for categorical variables and the Mann-Whitney U-test for continuous variables.

A mixed-effects model was applied to compare the postoperative changes in the JOA score and ODI between the OLF or CLF group and their matched control group. Finally, if there was a significant difference in the postoperative improvement in the clinical score, a multivariate linear regression model was applied. Variables with a significance of $\mathrm{p}<0.10$ in the univariate analysis and the presence of OLF or CLF were included as explanatory variables, and the recovery rate of the clinical score was set as the objective variable. Unstandardized partial regression coefficients (B), p values, and 95\% CIs were calculated. All analyses were performed using SPSS software (version 23; IBM Corp.). A value of $\mathrm{p}<0.05$ was considered statistically significant.

\section{Results}

\section{Overall Comparisons}

Among 4829 patients, 59 (1.2\%) had accompanying OLF and 39 (0.8\%) had accompanying CLF at the surgical level (Fig. 2). In the overall comparisons between patients with OLF, those with CLF, and those without OLF or CLF, there were significant differences in the average age $(\mathrm{p}<0.001)$ and distribution of diagnoses $(\mathrm{p}<0.001)$ (Table 1). In the post hoc analysis, patients with CLF were significantly older than those with OLF $(p=0.037)$ and without OLF or CLF ( $p<0.001)$. Furthermore, the ratio of patients with lumbar spinal stenosis was significantly higher among patients with OLF or CLF than among those without OLF or CLF $(p<0.05)$.

\section{Comparisons Between Patients With OLF and Matched Controls}

After adjusting for age, sex, preoperative JOA score, and diagnosis, patients with OLF $(\mathrm{n}=59)$ and matched 


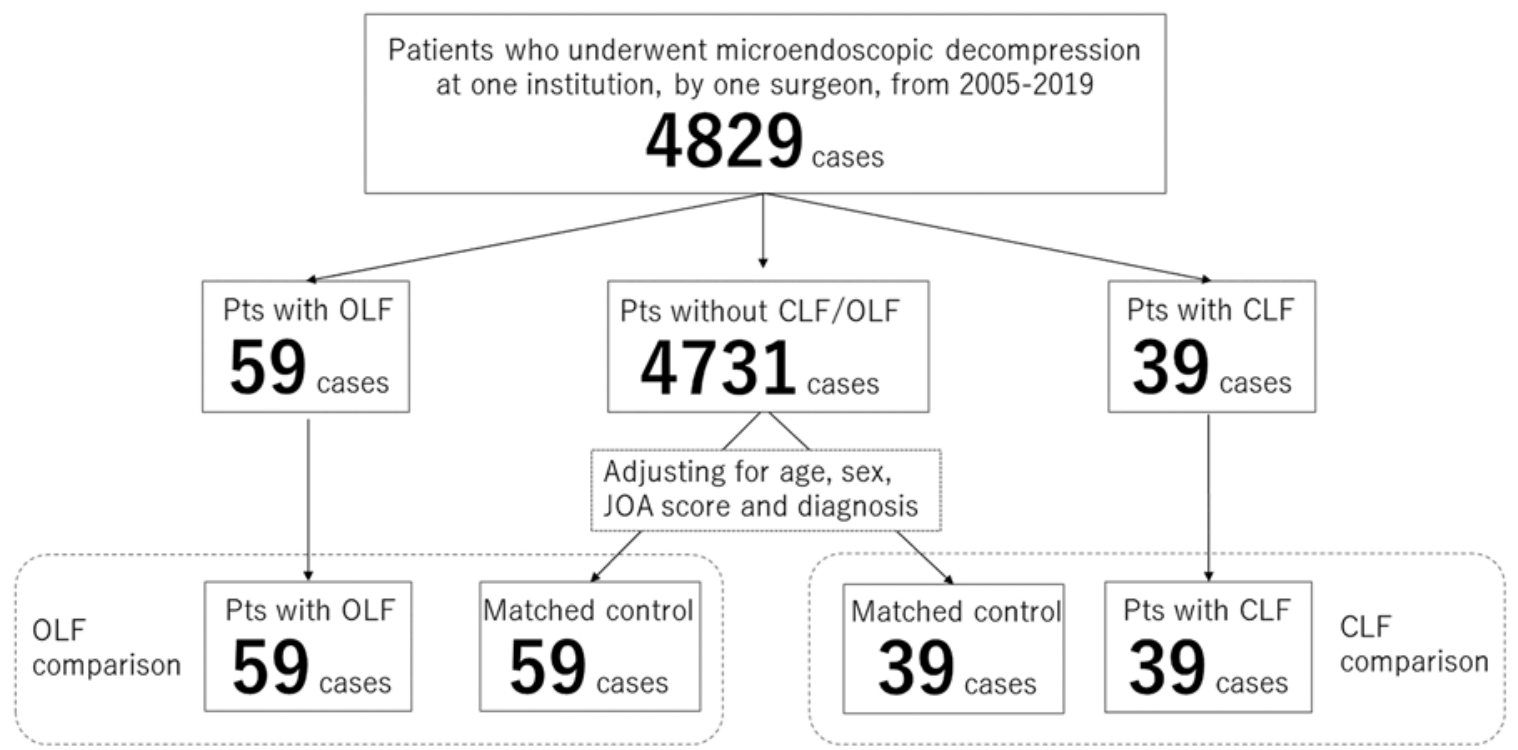

FIG. 2. Study design. Pts = patients.

controls $(n=59)$ were compared (Table 2). Patients with OLF had significantly higher weight $(\mathrm{p}=0.001)$, BMI ( $\mathrm{p}$ $=0.001)$, and incidence of preoperative motor weakness $(p=0.019)$ and longer operative time $(p<0.001)$ than the matched controls. However, the incidence of dural puncture and surgical outcomes such as the use of additional painkillers, presence of hematoma, and in-hospital days were not significantly different between the two groups. Although both groups showed significant improvements in clinical scores (relative to their preoperative score), the matched controls showed a significantly greater improvement in the JOA score than the patients with OLF ( $\mathrm{p}=$ 0.023 ; Table 3). In contrast, there was no significant difference in the improvement in the ODI between the two groups $(\mathrm{p}=0.134)$. Based on the results of the univariate analysis, the BMI and the presence of OLF, preoperative motor weakness, and diabetes were included as explanatory variables in the multivariate analysis, and the recovery rate of the JOA score was set as the objective variable. As a result, the presence of OLF was the only independent factor with significance $(\mathrm{p}<0.001$; Table 4$)$.

\section{Comparisons Between Patients With CLF and Matched Controls}

After adjusting for age, sex, preoperative JOA score, and diagnosis, patients with CLF $(\mathrm{n}=39)$ and matched controls $(\mathrm{n}=39)$ were compared (Table 5). There were no significant differences in the patients' background, co-

TABLE 1. Overall comparisons between patients with OLF, those with CLF, and those without OLF or CLF

\begin{tabular}{lcccr}
\hline \multicolumn{1}{c}{ Characteristic } & Pts w/ OLF & Pts w/ CLF & Pts w/o OLF or CLF & p Value \\
\hline No. of pts & 59 & 39 & 4731 & \\
\hline Age (yrs) & $60.7 \pm 15.2$ & $69.6 \pm 10.9$ & $56.1 \pm 17.7$ & $<0.001^{*}$ \\
\hline Sex (F/M) & $20 / 39$ & $20 / 19$ & $1654 / 3077$ & $0.102 \dagger$ \\
\hline Preop JOA score & $14.3 \pm 4.3$ & $13.8 \pm 4.8$ & $13.5 \pm 5.3$ & $0.473^{*}$ \\
\hline Preop ODI score & $39.7 \pm 16.8$ & $41.6 \pm 18.4$ & $44.5 \pm 19.4$ & $0.115^{*}$ \\
\hline Diagnosis & & & & \\
\hline Disc herniation & 6 & 1 & 2235 & $<0.001^{*}$ \\
\hline Lumbar spinal stenosis & 53 & 38 & 1852 & \\
\hline Foraminal stenosis & 0 & 0 & 131 & \\
\hline Other & 0 & 0 & 513 & \\
\hline Pis & & &
\end{tabular}

Pts $=$ patients

Values are presented as number of patients or mean \pm SD.

* One-way ANOVA.

† Chi-square test. 
TABLE 2. Comparisons between the OLF group and matched controls

\begin{tabular}{|c|c|c|c|}
\hline Characteristic & Pts w/ OLF & Matched Controls & $p$ Value \\
\hline No. of pts & 59 & 59 & \\
\hline Age (yrs) & $60.7 \pm 15.2$ & $61.5 \pm 14.9$ & $0.771^{*}$ \\
\hline $\operatorname{Sex}(F / M)$ & $20 / 39$ & $21 / 38$ & $0.694 \dagger$ \\
\hline Height (cm) & $163.7 \pm 8.0$ & $162.2 \pm 8.8$ & $0.309^{*}$ \\
\hline Weight (kg) & $70.8 \pm 12.9$ & $63.6 \pm 11.4$ & $0.001^{*}$ \\
\hline BMI & $26.4 \pm 4.4$ & $24.1 \pm 3.2$ & $0.001^{*}$ \\
\hline \multicolumn{4}{|l|}{ Comorbidity } \\
\hline Diabetes mellitus & 14 & 8 & $0.060 \dagger$ \\
\hline Hypertension & 17 & 18 & $1.000 \dagger$ \\
\hline Cardiac disease & 5 & 7 & $0.762 \dagger$ \\
\hline Respiratory disease & 3 & 4 & $1.000 \dagger$ \\
\hline Cerebrovascular disorders & 3 & 5 & $1.000 \dagger$ \\
\hline Diagnosis & & & $1.000 \dagger$ \\
\hline Disc herniation & 6 & 6 & \\
\hline Lumbar canal stenosis & 53 & 53 & \\
\hline \multicolumn{4}{|l|}{ Preop symptoms } \\
\hline Motor weakness (MMT $\leq 4)$ & 14 & 4 & $0.019 \dagger$ \\
\hline Bowel \& bladder dysfunction & 0 & 1 & $1.000 \dagger$ \\
\hline JOA score & $14.3 \pm 4.3$ & $14.0 \pm 4.5$ & $0.757^{*}$ \\
\hline ODI score & $39.7 \pm 16.8$ & $42.0 \pm 17.6$ & $0.468^{*}$ \\
\hline \multicolumn{4}{|l|}{ Surgical data } \\
\hline Total surgical time (mins) & $127 \pm 51$ & $97 \pm 37$ & $<0.001^{*}$ \\
\hline Surgical segments & $1.4 \pm 0.6$ & $1.3 \pm 0.5$ & $0.239^{*}$ \\
\hline Surgical time per segment (mins) & $94 \pm 28$ & $77 \pm 19$ & $<0.001^{*}$ \\
\hline Dural puncture & 3 & 1 & $0.619 \dagger$ \\
\hline Neurological deterioration & 0 & 0 & $1.000 \dagger$ \\
\hline In-hospital period (days) & $6.5 \pm 2.2$ & $6.9 \pm 4.3$ & $0.604^{*}$ \\
\hline Additional painkiller use & $0.9 \pm 0.9$ & $1.1 \pm 0.8$ & $0.452^{*}$ \\
\hline Hematoma on MRI & 4 & 3 & $1.000 \dagger$ \\
\hline
\end{tabular}

TABLE 3. Comparisons of the postoperative improvement in clinical scores

\begin{tabular}{|c|c|c|c|c|c|c|}
\hline Score & $\begin{array}{l}\text { Pts w/ OLF } \\
(n=59)\end{array}$ & $\begin{array}{c}\text { Matched Controls } \\
\quad(n=59)\end{array}$ & $\begin{array}{c}p \text { Value for } \\
\text { Global Change }\end{array}$ & $\begin{array}{l}\text { Pts w/ CLF } \\
\quad(n=39)\end{array}$ & $\begin{array}{l}\text { Matched Controls } \\
\qquad(n=39)\end{array}$ & $\begin{array}{c}\text { p Value for } \\
\text { Global Change }\end{array}$ \\
\hline JOA score & & & $0.023^{*}$ & & & $0.473^{*}$ \\
\hline Preop & $14.3 \pm 4.3$ & $14.0 \pm 4.5$ & & $13.8 \pm 4.8$ & $14.3 \pm 4.3$ & \\
\hline Postop & $24.8 \pm 3.1$ & $26.8 \pm 2.1$ & & $24.7 \pm 4.2$ & $26.1 \pm 2.0$ & \\
\hline ODI score & & & $0.134^{*}$ & & & $0.659^{*}$ \\
\hline Preop & $39.7 \pm 16.8$ & $42.0 \pm 17.6$ & & $41.6 \pm 18.4$ & $37.3 \pm 16.5$ & \\
\hline Postop & $9.8 \pm 11.2$ & $6.6 \pm 7.0$ & & $11.5 \pm 13.8$ & $6.7 \pm 9.1$ & \\
\hline
\end{tabular}

Values are presented as mean \pm SD.

* Mixed-effects model. 
TABLE 4. Results of the multivariate linear regression analysis

\begin{tabular}{lrrrr}
\hline & & & \multicolumn{2}{c}{$95 \% \mathrm{Cl}$} \\
\cline { 5 - 5 } Explanatory Variable* & \multicolumn{1}{c}{$\mathrm{B}$} & $\mathrm{p}$ Value & Lower Bound & Upper Bound \\
\hline OLF & -13.06 & $<0.001$ & -21.30 & -4.81 \\
\hline BMl & 0.25 & 0.651 & -0.86 & 1.37 \\
\hline Preop motor weakness & -4.53 & 0.394 & -15.06 & 5.99 \\
\hline Diabetes & -4.17 & 0.385 & -13.06 & 5.34 \\
\hline
\end{tabular}

$B=$ unstandardized partial regression coefficient.

* Objective variable: recovery rate of JOA score.

morbidities, preoperative symptoms, and surgical data between the two groups. Likewise, improvements in the JOA score and ODI did not significantly differ between the two groups (Table 3).

\section{Discussion}

Patients with OLF showed a higher BMI, higher incidence of preoperative motor weakness, longer operative time, and lower improvement in the JOA score than the

TABLE 5. Comparisons between the CLF group and matched controls

\begin{tabular}{|c|c|c|c|}
\hline Characteristic & Pts w/ CLF & Matched Controls & p Value \\
\hline No. of pts & 39 & 39 & \\
\hline Age (yrs) & $69.6 \pm 10.9$ & $69.2 \pm 10.5$ & $0.853^{*}$ \\
\hline Sex (F/M) & $20 / 9$ & $21 / 8$ & $1.000 \dagger$ \\
\hline Height (cm) & $158.3 \pm 10.7$ & $158.7 \pm 10.5$ & $0.893^{*}$ \\
\hline Weight (kg) & $61.6 \pm 12.2$ & $62.6 \pm 13.7$ & $0.734^{*}$ \\
\hline BMI & $24.4 \pm 3.5$ & $24.7 \pm 3.5$ & $0.773^{*}$ \\
\hline \multicolumn{4}{|l|}{ Comorbidity } \\
\hline Diabetes mellitus & 11 & 19 & $0.103 \dagger$ \\
\hline Hypertension & 18 & 24 & $0.256 \dagger$ \\
\hline Cardiac disease & 4 & 0 & $0.115 \dagger$ \\
\hline Respiratory disease & 3 & 4 & $1.000 \dagger$ \\
\hline Cerebrovascular disorders & 1 & 3 & $0.615 \dagger$ \\
\hline Diagnosis & & & $1.000 \dagger$ \\
\hline Disc herniation & 1 & 1 & \\
\hline Lumbar canal stenosis & 38 & 38 & \\
\hline \multicolumn{4}{|l|}{ Preop symptoms } \\
\hline Motor weakness (MMT $\leq 4)$ & 4 & 3 & $1.000 \dagger$ \\
\hline Bowel \& bladder dysfunction & 0 & 0 & $1.000 \dagger$ \\
\hline JOA score & $13.8 \pm 4.8$ & $14.3 \pm 4.3$ & $0.662 *$ \\
\hline ODI score & $41.6 \pm 18.4$ & $37.3 \pm 16.5$ & $0.298^{*}$ \\
\hline \multicolumn{4}{|l|}{ Surgical data } \\
\hline Total surgical time (mins) & $120 \pm 51$ & $121 \pm 60$ & $0.897^{*}$ \\
\hline Surgical segments & $1.5 \pm 0.7$ & $1.5 \pm 0.7$ & $0.866^{*}$ \\
\hline Surgical time per segment (mins) & $83 \pm 21$ & $83 \pm 28$ & $0.881^{*}$ \\
\hline Dural puncture & 0 & 2 & $0.494 \dagger$ \\
\hline Neurological deterioration & 1 & 1 & $1.000 \dagger$ \\
\hline In-hospital period (days) & $7.4 \pm 3.1$ & $6.5 \pm 1.8$ & $0.166^{*}$ \\
\hline Additional painkiller use & $1.1 \pm 0.8$ & $1.0 \pm 0.9$ & $0.788^{*}$ \\
\hline Hematoma on MRI & 3 & 3 & $1.000 \dagger$ \\
\hline
\end{tabular}


matched controls. However, only the presence of OLF was identified as an independent variable associated with the short-term recovery rate of the JOA score. In contrast, patients with CLF showed no significant differences from their matched controls in terms of preoperative, surgical, and postoperative data, including short-term improvements in the JOA score and ODI.

Okuda et al. ${ }^{24}$ reported a histological evaluation of the ligamentum flavum, which was resected en bloc from patients with degenerative lumbar disease. They found that 11 of 50 ligaments $(22 \%)$ showed calcification, with no specificity of the site of the lesions, appearing focally and dispersed in the ligamentum flavum. The incidence of CLF differed greatly between the report by Okuda et al. ${ }^{24}$ $(22 \%)$ and the present study ( $0.8 \%)$. This may be due to the detection tool used; namely, a histological evaluation can detect a small calcification that cannot be seen on CT images. However, the aim of the present study was to identify the outcomes and safety of surgical treatment for patients with CLF detectable on preoperative CT images.

There were no significant differences between patients with CLF and matched controls in terms of surgical complications and outcomes, including physician-assessed and patient-reported outcomes. These results may indicate that the surgical indications of minimally invasive posterior decompression for patients with degenerative lumbar disease accompanied by CLF can be considered to be the same as those for patients without CLF.

Interestingly, the improvement in the JOA score was significantly worse in patients with accompanying OLF than in matched controls. We first hypothesized that differences in the patients' background such as the higher $\mathrm{BMI}$ and higher incidence of motor weakness or diabetes in the patients with OLF influenced the surgical outcomes negatively. ${ }^{25,26}$ However, the multivariate regression model refuted this hypothesis; the existence of OLF was a significant factor associated with a poor recovery of the JOA score independently of BMI, motor weakness, and diabetes. Therefore, we now hypothesize that the biological mechanism of OLF might be a key factor in explaining this phenomenon. A histological evaluation of OLF demonstrated that the ossification is ascribable to the mechanism of endochondral ossification, with chondroid cells and cytokines from the enthesitis of the ligamentum flavum. ${ }^{24,27}$ These cells and signals can affect not only the ligamentum flavum but also the dura, resulting in degenerative changes such as ossification. Indeed, several studies have demonstrated that up to half of the patients with OLF have intraoperative evidence of dural ossification..$^{28,29}$ Further research is needed to validate the current hypothesis. In addition, the clinical outcomes of other surgical methods such as open decompression or decompression with interbody fusion should be evaluated and compared with the outcomes of microendoscopic decompression to establish the appropriate treatment strategy for patients with lumbar spinal stenosis with OLF.

There are several limitations to the present study. First, the diagnoses of OLF and CLF in this study were based on the evaluation of preoperative CT images, but not histological findings and findings during surgery. Second, our standardized postoperative care, including the in-hospital period, is allowed in the Japanese system but is not practical under the insurance system in several countries, such as the United States. However, we believe that current results also provide some beneficial messages for physicians in such countries. Third, clinical scores were only assessed 3 months postoperatively. Although some reports have demonstrated that the short-term improvement of the clinical score after minimally invasive decompression surgery could be maintained up to 2 years postoperatively, ${ }^{30,31}$ it is uncertain whether such results could be applied to the uncommon pathology found in OLF and CLF cases. Hence, current results must be validated in future studies with long-term follow-up. Finally, although the surgical criteria at our institution did not change due to the presence of OLF or CLF, the retrospective nature of the study renders it difficult to exclude a selection bias completely.

To overcome such limitations, large-scale prospective and randomized surgical procedure studies with a longterm follow-up of patients with OLF and CLF should be designed with an adequate number of patients. However, the strengths of the present study are that all cases were treated at a single institution, which can exclude variations in surgical indications, surgical methods, surgical skills, and pre- and postoperative care-and detailed preoperative data were provided. In addition, the number of patients in the present study is the largest reported to date; this is a strength of our study, because OLF and CLF that can be identified with preoperative CT are relatively rare conditions (representing approximately $1.0 \%$ of all patients with lumbar degenerative disease). Therefore, despite the abovementioned limitations, we believe that the findings of the current study can provide beneficial knowledge and aid physicians in developing an effective and safe surgical strategy for the treatment of patients with OLF or CLF.

\section{Conclusions}

Although the perioperative surgical outcomes, including the surgical complications, in-hospital period, and the use of painkillers, did not significantly differ, the shortterm improvement in the JOA score was significantly lower in patients with degenerative lumbar disease accompanied by OLF than in the matched control group. Furthermore, the presence of OLF was significantly and independently associated with a poor recovery in the JOA score. In contrast, there were no significant differences in the short-term improvement in clinical scores and perioperative outcomes between patients with CLF and their matched control group. Thus, the surgical indications of minimally invasive posterior decompression for patients with CLF can be the same as those for patients without CLF. Meanwhile, the indications for patients with OLF should be further investigated in future studies, including the other surgical methods.

\section{Acknowledgments}

We thank the individuals who contributed to the study or manuscript preparation but do not fulfill all the criteria of authorship.

\section{References}

1. Mori K, Kasahara T, Mimura T, et al. Prevalence, distribu- 
tion, and morphology of thoracic ossification of the yellow ligament in Japanese: results of CT-based cross-sectional study. Spine (Phila Pa 1976). 2013;38(19):E1216-E1222.

2. Shepard NA, Shenoy K, Cho W, D Sharan A. Extensive ossification of the ligamentum flavum treated with triple stage decompression: a case report. Spine J. 2015;15(4):e9-e14.

3. Guo JJ, Luk KD, Karppinen J, et al. Prevalence, distribution, and morphology of ossification of the ligamentum flavum: a population study of one thousand seven hundred thirty-six magnetic resonance imaging scans. Spine (Phila Pa 1976). 2010;35(1):51-56.

4. Kurihara A, Tanaka Y, Tsumura N, Iwasaki Y. Hyperostotic lumbar spinal stenosis. A review of 12 surgically treated cases with roentgenographic survey of ossification of the yellow ligament at the lumbar spine. Spine (Phila Pa 1976). 1988; 13(11):1308-1316.

5. Gao R, Yuan W, Yang L, et al. Clinical features and surgical outcomes of patients with thoracic myelopathy caused by multilevel ossification of the ligamentum flavum. Spine $J$. 2013;13(9):1032-1038.

6. Ruiz Santiago F, Alcázar Romero PP, López Machado E, García Espona MA. Calcification of lumbar ligamentum flavum and facet joints capsule. Spine (Phila Pa 1976). 1997; 22(15):1730-1735.

7. Khan MH, Smith PN, Donaldson WF III. Acute quadriparesis caused by calcification of the entire cervical ligamentum flavum in a white female-report of an unusual case and a brief review of the literature: case report. Spine (Phila Pa 1976). 2005;30(22):E687-E691.

8. Yayama T, Baba H, Furusawa N, et al. Pathogenesis of calcium crystal deposition in the ligamentum flavum correlates with lumbar spinal canal stenosis. Clin Exp Rheumatol. 2005; 23(5):637-643.

9. Telfeian AE, Veeravagu A, Oyelese AA, Gokaslan ZL. A brief history of endoscopic spine surgery. Neurosurg Focus. 2016;40(2):E2.

10. Minamide A, Yoshida M, Yamada H, et al. Endoscope-assisted spinal decompression surgery for lumbar spinal stenosis. $J$ Neurosurg Spine. 2013;19(6):664-671.

11. Pao JL, Chen WC, Chen PQ. Clinical outcomes of microendoscopic decompressive laminotomy for degenerative lumbar spinal stenosis. Eur Spine J. 2009;18(5):672-678.

12. Fujii K, Abe T, Funayama T, et al. Lateral lumbar interbody fusion for ossification of the yellow ligament in the lumbar spine: first reported case. Case Rep Orthop. 2017;2017: 3404319 .

13. Katz JN, Stucki G, Lipson SJ, et al. Predictors of surgical outcome in degenerative lumbar spinal stenosis. Spine (Phila Pa 1976). 1999;24(21):2229-2233.

14. Yamashita K, Ohzono K, Hiroshima K. Five-year outcomes of surgical treatment for degenerative lumbar spinal stenosis: a prospective observational study of symptom severity at standard intervals after surgery. Spine (Phila Pa 1976). 2006; 31(13):1484-1490.

15. Rubin DB. Propensity score methods. Am J Ophthalmol. 2010;149(1):7-9.

16. Rubin DB. The design versus the analysis of observational studies for causal effects: parallels with the design of randomized trials. Stat Med. 2007;26(1):20-36.

17. Minamide A, Yoshida M, Simpson AK, et al. Minimally invasive spinal decompression for degenerative lumbar spondylolisthesis and stenosis maintains stability and may avoid the need for fusion. Bone Joint J. 2018;100-B(4):499-506.

18. al-Orainy IA, Kolawole T. Ossification of the ligament flavum. Eur J Radiol. 1998;29(1):76-82.
19. Sato R, Takahashi M, Yamashita Y, et al. Calcium crystal deposition in cervical ligamentum flavum: CT and MR findings. J Comput Assist Tomogr. 1992;16(3):352-355.

20. Fujiwara A, Kobayashi N, Saiki K, et al. Association of the Japanese Orthopaedic Association score with the Oswestry Disability Index, Roland-Morris Disability Questionnaire, and Short-Form 36. Spine (Phila Pa 1976). 2003;28(14): 1601-1607.

21. Fairbank JC, Couper J, Davies JB, O’Brien JP. The Oswestry low back pain disability questionnaire. Physiotherapy. 1980; 66(8):271-273.

22. Hirabayashi K, Watanabe K, Wakano K, et al. Expansive open-door laminoplasty for cervical spinal stenotic myelopathy. Spine (Phila Pa 1976). 1983;8(7):693-699.

23. Haberman SJ. The analysis of residuals in cross-classified tables. Biometrics. 1973;29(1):205-220.

24. Okuda T, Baba I, Fujimoto Y, et al. The pathology of ligamentum flavum in degenerative lumbar disease. Spine (Phila $P a$ 1976). 2004;29(15):1689-1697.

25. Takenaka S, Aono H. Prediction of postoperative clinical recovery of drop foot attributable to lumbar degenerative diseases, via a Bayesian network. Clin Orthop Relat Res. 2017; 475(3):872-880.

26. Iizuka Y, Iizuka H, Tsutsumi S, et al. Foot drop due to lumbar degenerative conditions: mechanism and prognostic factors in herniated nucleus pulposus and lumbar spinal stenosis J Neurosurg Spine. 2009;10(3):260-264.

27. Li B, Guo S, Qiu G, et al. A potential mechanism of dural ossification in ossification of ligamentum flavum. Med Hypotheses. 2016;92:1-2.

28. Muthukumar N. Dural ossification in ossification of the ligamentum flavum: a preliminary report. Spine (Phila Pa 1976). 2009;34(24):2654-2661.

29. Yu L, Li B, Yu Y, et al. The relationship between dural ossification and spinal stenosis in thoracic ossification of the ligamentum flavum. J Bone Joint Surg Am. 2019;101(7):606-612.

30. Youn MS, Shin JK, Goh TS, Lee JS. Predictors of clinical outcome after endoscopic partial facetectomy for degenerative lumbar foraminal stenosis. World Neurosurg. 2019;126: e1482-e1488.

31. Ikuta K, Arima J, Tanaka T, et al. Short-term results of microendoscopic posterior decompression for lumbar spinal stenosis. Technical note. J Neurosurg Spine. 2005;2(5):624-633.

\section{Disclosures}

The authors report no conflict of interest concerning the materials or methods used in this study or the findings specified in this paper.

\section{Author Contributions}

Conception and design: Tamai, Shimada. Acquisition of data: Tamai, Kaneda, Iwamae, Katsuda. Analysis and interpretation of data: Tamai, Iwamae, Terai. Drafting the article: Tamai, Kaneda, Katsuda, Shimada. Critically revising the article: Kaneda, Terai, Nakamura. Reviewed submitted version of manuscript: Kaneda, Iwamae, Terai, Katsuda, Shimada, Nakamura. Study supervision: Nakamura.

\section{Correspondence}

Koji Tamai: Osaka City University Graduate School of Medicine, Osaka, Japan.koji.tamai@msic.med.osaka-cu.ac.jp. 